Sammlung Metzler

Band 250 


\section{Hermann Korte}

\section{Geschichte der deutschen Lyrik seit 1945}

J.B. Metzlersche Verlagsbuchhandlung Stuttgart 
CIP-Titelaufnahme der Deutschen Bibliothek

\author{
Korte, Hermann: \\ Geschichte der deutschen Lyrik seit 1945 \\ / Hermann Korte. \\ - Stuttgart : Metzler, 1989 \\ (Sammlung Metzler ; Bd. 250) \\ ISBN 978-3-476-10250-8 \\ ISBN 978-3-476-03952-1 (eBook) \\ DOI 10.1007/978-3-476-03952-1
}

NE: GT

Dieses Werk einschließlich aller seiner Teile ist urheberrechtlich geschützt. Jede Verwertung außerhalb der engen Grenzen des Urheberrechtsgesetzes ist ohne Zustimmung des Verlages unzulässig und strafbar. Das gilt insbesondere für Vervielfältigungen, Übersetzungen, Mikroverfilmungen und die Einspeicherung und Verarbeitung in elektronischen Systemen.

(C) 1989 Springer-Verlag GmbH Deutschland Ursprünglich erschienen bei J.B. Metzlersche Verlagsbuchhandlung und Carl Ernst Poeschel Verlag GmbH in Stuttgart 1989 


\section{Inhaltsverzeichnis}

Vorwort $\ldots \ldots \ldots \ldots \ldots \ldots \ldots \ldots \ldots \ldots \ldots \ldots \ldots$ IX

I. Kontinuitäten. Lyrik zwischen 1945 und 1949 ... 1

1. 1945 - Weder "Kahlschlag « noch "Stunde Null« .... 1

2. Poetische Kontrafakturen unnd ihre Grenzen. $»$ Trümmerlyrik $\ldots \ldots \ldots \ldots \ldots \ldots \ldots \ldots . . \quad 8$

3. Zwischen Konvention und Irritation. Paradigmen des Traditionalismus ....................... 17

II. Tradition und Artistik. Die fünfziger Jahre ...... 30

1. Universalchiffre »Natur«. Das Naturgedicht der fünfziger Jahre $\ldots \ldots \ldots \ldots \ldots \ldots \ldots \ldots \ldots \ldots, 30$

2. Poesie als Widerstand. Hermetische Tendenzen .... 44

3. Inszenierungen des lyrischen Ichs. Zur Poetologie des Nachkriegsgedichts .................... 60

4. Rückkehr zum Experiment. Anfänge der Konkreten Poesie ............................ 71

III. Umbrüche. Die sechziger Jahre ............. 82

1. Vor dem Verstummen? Späthermetismus und Naturlyrik in den sechziger Jahren $\ldots \ldots \ldots \ldots \ldots . \quad 82$

2. Die »Entdeckung der Wirklichkeit«. Paradigmenwechsel in der Lyrik ............. 100

3. Das »Gedicht im Handgemenge«. Lyrik und LyrikDebatten um 1968 ........................ 117

4. Die zweite Generation. Neue Formationen in der DDR-Lyrik ....................... 126

5. Manifestation und Kalkül. Möglichkeiten und Grenzen Konkreter Poesie .................. 136

IV. Lädierte Utopien. Die siebziger Jahre .......... 144 1. »Alltagslyrik«. Anspruch und Wirklichkeit eines Genres ............................ 144

2. Erfahrungen nach 1968. Kontroversen um neue Subjektivität . ....................... 157

3. Utopien im Widerspruch. Tendenzen der 70er Jahre . 166 
V. Auf dem Weg zur Postmoderne? Lyrik seit 1980 . 185

1. Nach der sneuen Subjektivität $\longleftarrow$ Die Wiederkehr der Form ............................ 185

2. Weder Postmoderne noch Neo-Avantgarde. Tendenzen seit 1980 ....................... 195

VI. Bibliographie ........................ 208

1. Bibliographien, Handbücher, Lexika . ........... 208

2. Auswahl-Chronologie: Gedichtbände, Anthologien und Sammlungen 1945-1985 ............... 209

3. Gesamtausgaben, Gesammelte Werke, Werke in Auswahl .......................... 223

4. Literatur zur Lyrik nach 1945 . . . . . . . . . . . . 225

Namenregister ........................... 239 


\section{Abkürzungen}

Akz. Akzente. Zeitschrift für Literatur

Basis Basis. Jahrbuch für deutsche Gegenwartsliteratur

DK Duitse Kroniek

DU Der Deutschunterricht

DVjs Deutsche Vierteljahrsschrift für Literaturwissenschaft und Geistesgeschichte

Et. Germ. Etudes germaniques

GR The German review

GRM Germanisch-Romanische Monatsschrift

JbDDR Jahrbuch zur Literatur in der DDR

JbSG Jahrbuch der deutschen Schillergesellschaft

LJb Literaturwissenschaftliches Jahrbuch N. F.

LM Literaturmagazin

LuK Literatur und Kritik

LWU Literatur in Wissenschaft und Unterricht

MGS Michigan Germanic Studies

NDH Neue deutche Hefte

NDL Neue deutsche Literatur

NR Neue Rundschau

RG Recherches germaniques

SptZ Sprache im technischen Zeitalter

SuF Sinn und Form

TeKo Text und Kontext

TuK Text und Kritik. Zeitschrift für Literatur

WB Weimarer Beiträge 


\section{Zur Zitierweise}

Die zitierte Literatur wird über Zahlen nachgewiesen, welche die entsprechende Nummer in der Bibliographie (vg. Kapitel VI) sowie die Seitenzahl des jeweiligen Titels angeben. 


\section{Vorwort}

Der vorliegende Band will in die Geschichte der deutschen Lyrik nach 1945 einführen und bibliographisches Material zu einem bislang wenig überschaubaren Thema sichern.

Die Abhandlung ist an Prämissen geknüpft, die sich aus ihrem Gegenstand selbst ergeben. So gilt der intendierte Überblick einem historischen Prozeß, der in die Gegenwart hineinreicht und von ihr mit nur geringem Abstand beurteilt werden kann. Auswahl und Systematisierung, Kritik und Wertung sind notwendig an diese hermeneutische Situation gebunden. Es wird daher auf eine Konstruktion epochaler Zäsuren ebenso verzichtet wie auf jenen fragwürdigen Versuch die Lyrik nach 1945 in Schulen und Richtungen, Gattungen und Genres zu gliedern. Statt dessen wählt die vorliegende Arbeit den chronologischen Rekurs : nicht weil die einzelnen Dekaden geschichtliche Abschnitte sui generis bilden, sondern weil der zunächst äußere, am historischen Verlauf orientierte Gliederungsmodus einen ersten Zugang zum Gegenstand erlaubt. Der historische Prozeß freilich, von den späten 40er bis zu den 80er Jahren entfaltet, soll gerade im diachronen Schnitt keine lineare Kontinuität und schon gar keine teleologische Zielperspektive suggerieren. Vielmehr gilt es die Bruchstellen und Diskontinuitäten, ja die widersprüchliche Vielfalt der Lyrik nach 1945 zu bestimmen, indem ihre geschichtliche Signatur offengelegt wird.

Eine solche Untersuchung ist keine Summe aus einzelnen Werkgeschichten, schon gar keine Synopse von Dichterbiographien und Lebensläufen. Das einzelne Gedicht, der einzelne Gedichtband eines Autors werden zum Element eines historischen Diskurses, zum Paradigma einer Darstellung, die ihre Auswahl und Akzente auf die Gattungsgeschichte selbst richtet und nach dem je historischen Ort einzelner Werke fragt. Anders formuliert: Das Buch gibt keinen abrißartigen Kommentar zur Lyrik Paul Celans, aber dessen Dichtung wird zum exzeptionellen Beispiel einer Nachkriegslyrik, deren Geschichte sie bis heute hin mitgeschrieben hat. Es versteht sich vor diesem Hintergrund, daß die geringe Distanz zum Gegenstand nicht eine Beliebigkeit und Subjektivität in Urteil und Wert- 
maßstab rechtfertigt, sondern deren umso sorgfältigere hermeneutische Reflexion.

Der Gegenstand der Abhandlung ist ein historisches Thema. Es wird sich sogar zeigen, daß der vielzitierte Topos vom sschnellen Altern $<$ der Gegenwartsliteratur einige Berechtigung hat : daß in der Lyrik seit 1945 Richtungen und Trends längst ihre Geschichte haben, ad acta gelegt und sogar schon vergessen sind, und daß von $\operatorname{der}$ Geschichte der Nachkriegslyrik zu sprechen ein Wagnis ist, da eine Tendenz zur beziebungslosen Gleichzeitigkeit von Literaturen nach 1945 sich verstärkt hat. Indes kommt sie gerade nicht im schon historisch gewordenen Streit um eine, zwei oder mehrere deutsche Literaturen zum Ausdruck. Und daß die Lyrik der DDR ihren prägenden Anteil am Nachkriegsgedicht hat, dazu liefert die Darstellung aus allen Dekaden anschauliche Beispiele.

Die Geschichte der Nachkriegslyrik schließlich ist eine Konstruktion des Literarhistorikers: entworfen aus seinen Fragen, seinen Akten des Gliederns und Ordnens, seinen Erkenntnisinteressen. Wenn im Verlaufe der Darstellung deutlich wird, daß es den einen roten Faden in der Geschichte der Lyrik nach 1945 nicht gibt, sondern sie ein Kaleidoskop widersprüchlicher, abgebrochener, ja divergenter Geschichten ist : voller historischer 'Unordnung, dann hat der vorliegende Band sein Ziel erreicht.

Münster, im November 1988

Hermann Korte 